\title{
A pH-Sensitive Injectable Nanoparticle Composite Hydrogel for Anticancer Drug Delivery
}

\author{
Yuanfeng Ye and Xiaohong Hu \\ School of Material Engineering, Jinling Institute of Technology, Nanjing 211169, China \\ Correspondence should be addressed to Xiaohong Hu; hxh@jit.edu.cn
}

Received 26 November 2015; Accepted 24 January 2016

Academic Editor: Ester Vazquez

Copyright ( 2016 Y. Ye and X. Hu. This is an open access article distributed under the Creative Commons Attribution License, which permits unrestricted use, distribution, and reproduction in any medium, provided the original work is properly cited.

\begin{abstract}
According to previous reports, low pH-triggered nanoparticles were considered to be excellent carriers for anticancer drug delivery, for the reason that they could trigger encapsulated drug release at mild acid environment of tumor. Herein, an acidsensitive $\beta$-cyclodextrin derivative, namely, acetalated- $\beta$-cyclodextrin (Ac- $\beta$-CD), was synthesized by acetonation and fabricated to nanoparticles through single oil-in-water $(\mathrm{o} / \mathrm{w})$ emulsion technique. At the same time, camptothecin (CPT), a hydrophobic anticancer drug, was encapsulated into Ac- $\beta$-CD nanoparticles in the process of nanoparticle fabrication. Formed nanoparticles exhibited nearly spherical structure with diameter of $209 \pm 40 \mathrm{~nm}$. The drug release behavior of nanoparticles displayed $\mathrm{pH}$ dependent changes due to hydrolysis of $A c-\beta-C D$. In order to overcome the disadvantages of nanoparticle and broaden its application, injectable hydrogels with Ac- $\beta$-CD nanoparticles were designed and prepared by simple mixture of nanoparticles solution and graphene oxide (GO) solution in this work. The injectable property was confirmed by short gelation time and good mobility of two precursors. Hydrogels were characterized by dynamic mechanical test and SEM, which also reflected some structural features. Moreover, all hydrogels underwent a reversible sol-gel transition in alkaline environment. Finally, the results of in vitro drug release profile indicated that hydrogel could control drug release or bind drug inside depending on the $\mathrm{pH}$ value of released medium.
\end{abstract}

\section{Introduction}

Given the fact that the $\mathrm{pH}$ value in normal tissue is about 7.4, the $\mathrm{pH}$ value in infectious and inflammatory sites is about 6.5 , and the $\mathrm{pH}$ values in tumor tissue are lower than 6.5 , low $\mathrm{pH}$-triggered nanoparticles have received much concern in anticancer drug delivery system, aiming at achieving better efficacy and avoiding side effects [1-3]. In view of good processability and degradation, synthesized ester polymers or copolymers were primary materials for low $\mathrm{pH}$-triggered nanoparticles $[4,5]$. But as for natural cyclodextrins (CDs), proven effective materials to control drug release, they were seldom fabricated to be low $\mathrm{pH}$-triggered nanoparticles alone on account of their poor processability and no $\mathrm{pH}$-sensitive property. Recently, the emerging of acetalated CDs (Ac-CDs) changed this condition [6]. Ac-CD could be prepared to be low $\mathrm{pH}$-triggered nanoparticles by microemulsion method $[1-3,7-9]$. As far as the application of nanoparticles was concerned, they were mainly used as targeted anticancer drug carrier, which meant that nanoparticles must get through all tiny blood capillary before they reached the targeted site [13]. However, the size of Ac-CD nanoparticles was not small enough to get through the tiniest blood capillary according to previous report. Furthermore, they as $1 \mathrm{D}$ material were hardly fixed to targeted sites for some period even if they were injected to that site. Besides the above-mentioned disadvantages, another could not be neglected; that is, tedious centrifugal separation process in microemulsion method not only deteriorated the output of nanoparticle but also resulted in some broken nanoparticles with lost drug. Therefore, it has great significance to establish a 3D material using Ac-CD nanoparticle solution without getting rid of emulsifier, aimed at anticancer drug delivery.

Hydrogel, the semisolid material, which is composed of hydrophilic polymer network and enormous embedded water, has been intensively investigated. The solid-liquid structure endows hydrogel with a number of flexible properties like adjustable swelling property, stimuli-responsive 
ability, and biocompatibility [10-13]. Besides these merits, injectable hydrogel has advantages of minimally invasive operation and filling irregular defects of tissues [12-14]. In this sense, injectable hydrogel is increasingly attractive in the field of tissue engineering and drug delivery [10-14]. The driving force of injectable hydrogel includes chemical crosslinking, polymerization in situ, electrostatic interaction, and host-guest interaction [14]. Based on the facts that chemical methods have some disadvantages of toxicity, severe reaction condition, physical methods are interesting approaches for injectable hydrogel investigation, though the physical driving force is often not strong enough to support hydrogel for long-term use. Herein, we attempted to design an injectable hydrogel based on Ac-CD nanoparticle solution using a physical driving force for the hydrophobic drug delivery.

Recently, it was reported that graphene oxide (GO) and polyvinyl alcohol (PVA) could self-assemble into supermolecular hydrogel in aqueous solution due to the strong interfacial interaction between these two components [1518]. GO, a member of graphene family, also possessed excellent properties of graphene like mechanical properties, large surface area, low coefficient of thermal expansion, and very high aspect ratio $[13,19]$. When it came to graphene family, the newly interesting materials displayed great potential in the drug delivery field, especially for poorly soluble drug delivery, on account of its typical two-dimensional $\pi$ conjugated structure [13]. For this reason, a number of hydrogels and nanocarriers made by graphene family material became new favorites of researchers [20-22]. Since PVA was a main emulsifier in the process of nanoparticle preparation, injectable hydrogels were assembled by supermolecular interaction between GO and PVA in this work. Moreover, the properties of both low $\mathrm{pH}$-triggered nanoparticles and injectable hydrogel were investigated in the work.

Camptothecin (CPT), a hydrophobic anticancer drug, has limited curative effect on account of slight solubility in aqueous environment, which results in intensive research on CPT modifications to improve its solubility in aqueous solution $[8,13,23]$. Among these modifications, the addition of solubilizers like CDs and their derivatives is an effective and efficient method $[13,23]$. So it is inferred that Ac$\beta-\mathrm{CD}$, a CD derivative, was also a kind of ideal carrier material. Hence, CPT was chosen as a model drug and encapsulated in Ac- $\beta$-CD nanoparticle during the fabrication of nanoparticles in the work.

\section{Experimental Section}

2.1. Materials. $\beta$-Cyclodextrin, 2-methoxypropene, pyridinium 4-toluenesulfonate, dimethyl sulfoxide (DMSO), and dichloromethane (DCM) were obtained from Shanghai Chemical Industries Co., Ltd. (China). Poly(vinyl alcohol) (PVA) (87-89 mol\% hydrolyzed, CPS: 4.6-5.4) was obtained from Aladdin (Shanghai) Co., Ltd. (China). Camptothecin (CPT) and dialysis tube $\left(M_{w}: 8000-14000\right)$ were purchased from Sigma. Graphene oxide (GO, purity: 99 wt\%; diameter: $500 \mathrm{~nm}-5 \mu \mathrm{m}$; thickness: $0.8-1.2 \mathrm{~nm}$ ) was purchased from Nanjing Pioneer Nano-Tech Co. (China), which was synthesized by Hummers method with $99 \%$ single layer ratio.
The XPS result indicated that $\mathrm{C}$ atomic concentration is $71.23 \%$ and $\mathrm{O}$ atomic concentration is $28.77 \%$. The other characterizations were shown in Figure 1. These results were provided by Nanjing Pioneer Nano-Tech Co.

2.2. Synthesis of $A c-\beta-C D$. Acetonation was conducted by the reaction between 2-methoxypropene and hydroxyl groups using pyridinium p-toluenesulfonate as a catalyst [6]. Briefly, $\beta$-CD $(1.03 \mathrm{mmol})$ and pyridinium $\mathrm{p}$ toluenesulfonate $(0.1 \mathrm{mmol})$ were added into 3 -mouth flask, filled with $\mathrm{N}_{2}$, into which $20 \mathrm{~mL}$ anhydrous DMSO and 2methoxypropene ( $42 \mathrm{mmol}$ ) were, respectively, dropped. The whole reaction was performed under anhydrous oxygenfree environment at $30^{\circ} \mathrm{C}$. After the mixture was stirred for $6 \mathrm{~h}$, the acetalated $\beta$-CD (Ac- $\beta$-CD) was precipitated from basic water $\left(\mathrm{pH}=7.5\right.$, adjusted by $\left.\mathrm{NH}_{3}\right)$, collected by filtration, then washed with basic water, and lastly lyophilized to a white powder. Ac- $\beta$-CD was characterized by infrared spectroscopy (IR, IS10). Moreover, the solubility of product was tested in water with different $\mathrm{pH}$ value.

\subsection{Fabrication and Characterization of Nanoparticle. CPT} encapsulated nanoparticles were prepared by single emulsion method using DCM as solvent, DMSO as cosolvent for CPT, and water as dispersing agent. Briefly, 5\% (w/v) $100 \mu \mathrm{L}$ CPT/DMSO solution was mixed with $10 \% 1 \mathrm{~mL}$ Ac- $\beta$-CD/DCM solution to obtain homogeneous solution, which was emulsified via probe sonication ( $15 \mathrm{w}, 1 \mathrm{~min})$ into $10 \mathrm{~mL}$ of $1 \%$ PVA aqueous solution. The obtained emulsion was immediately added into $20 \mathrm{~mL}$ of $0.3 \%$ PVA aqueous solution after probe sonication. The solvent evaporation lasted for $10 \mathrm{~h}$ under magnetic stirring. Then, CPT-loaded Ac- $\beta$-CD nanoparticles were collected by centrifugation (14000 rpm, $10 \mathrm{~min}$ ), washed several times with basic water, and lyophilized. These nanoparticles were characterized by scanning electron microscope (SEM, S8100).

In vitro drug release study was carried out under $\mathrm{PBS}$ buffer with different $\mathrm{pH}$ value (adjusted by $\mathrm{HCl}$ or $\mathrm{NH}_{3}$ ). Briefly, about $17.0 \mathrm{mg}$ lyophilized CPT-loaded nanoparticles were dispersed in $4.0 \mathrm{~mL}$ release medium. At appropriate intervals, $1.0 \mathrm{~mL}$ release medium was withdrawn after centrifugation at $14000 \mathrm{rpm}$ for $10 \mathrm{~min}$, and the same volume of fresh medium was added. The released CPT was quantified by ultraviolet spectrophotometric method using UV spectroscopy (Cary 50) at $360 \mathrm{~nm}$.

2.4. Characterization of Injectable Hydrogel. The injectable composite hydrogel was prepared by simple mixture of GO solution and PVA solution, which are described as follows. $642 \mathrm{~mL}$ GO dispersion $(7.8 \mathrm{mg} / \mathrm{mL})$ was added into $358 \mathrm{~mL}$ above-mentioned PVA solution with prepared nanoparticles, and the mixture was shaken violently until a gel was formed. The formation of the gel was confirmed by a tube inversion method, so did the gelation time of the physical gel. The final hydrogel was further treated by sonication for $20 \mathrm{~min}$ to make the gelation process complete. The final hydrogels were characterized by rheological measurement in a parallel plate mode using a strain-controlled rheometer (MCR102). 


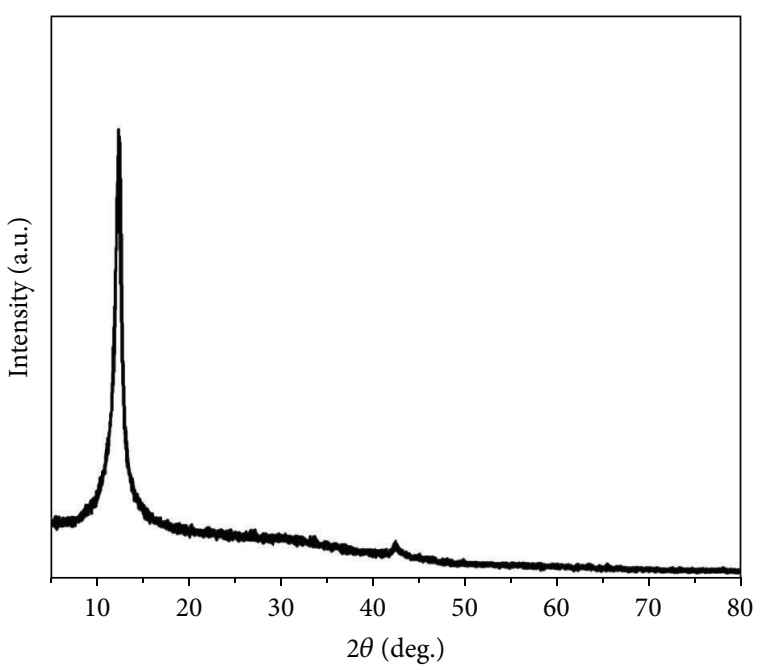

(a)

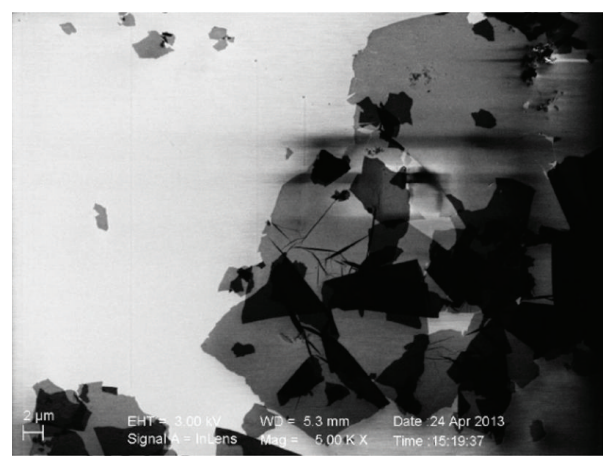

(b)

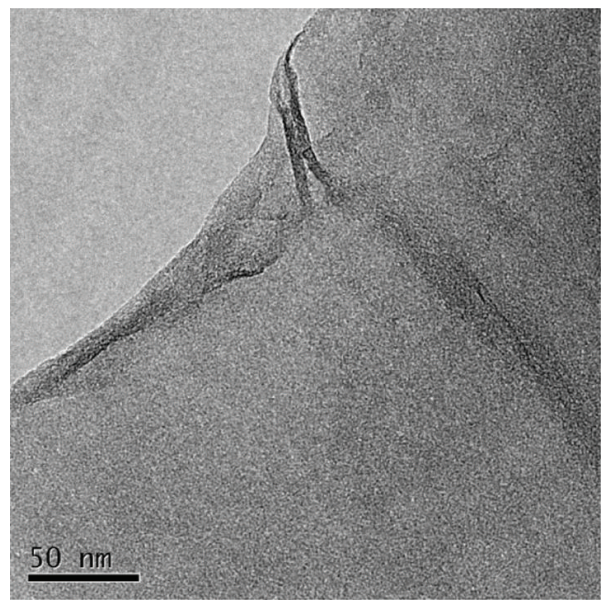

(c)

FIGURE 1: (a) XRD result of GO. (b) SEM images of GO. (c) TEM images of GO.

Dynamic oscillatory mode (compression mode) was used to measure the storage and loss moduli. All tests were performed at $37^{\circ} \mathrm{C}$ with a fixed strain of $1 \%$. Moreover, lyophilized hydrogels were characterized by SEM (S8100).

In vitro drug release study was carried out under PBS buffer with different $\mathrm{pH}$ value (adjusted by $\mathrm{HCl}$ or $\mathrm{NH}_{3}$ ). Briefly, $400 \mu \mathrm{L}$ hydrogel was sealed in dialysis tube, which was further dispersed in $4.0 \mathrm{~mL}$ release medium. At appropriate intervals, $1.0 \mathrm{~mL}$ release medium was withdrawn, and the same volume of fresh medium was added. The released CPT was quantified by ultraviolet spectrophotometric method using UV spectroscopy (Cary 50) at $360 \mathrm{~nm}$.

\section{Results and Discussion}

3.1. CPT-Loaded Ac- $\beta$-CD Nanoparticles. Acetonation, a temporary protection reaction of diol functions, was used to prepare acid-sensitive $\beta$-CD (Figure 2(a)), which was confirmed by IR spectroscopy in Figure 2(b). In the IR spectrum of Ac- $\beta-C D$, the appearance of absorbance bands at 2991, 2937, and $1377 \mathrm{~cm}^{-1}$ that are characteristic bands for $-\mathrm{C}\left(\mathrm{CH}_{3}\right)_{2}$ group, as well as the significant attenuated absorption at $3427 \mathrm{~cm}^{-1}$ due to hydroxyl group, suggested the formation of acetals. The dissolubility experiments showed that Ac- $\beta$-CD was stable in neutral and alkaline water $(\mathrm{pH}>$ 7 ) and dissolved in mild acidic water $(\mathrm{pH}<6)$, which confirmed the $\mathrm{pH}$-responsive property of Ac- $\beta$-CD.

Given that Ac- $\beta$-CD could be easily dissolved in common organic solvents like ethanol, DCM, acetone, acetonitrile, and THF, a simple approach, emulsion solvent evaporation technique, was assumed to prepare Ac- $\beta$-CD nanoparticles conveniently. It is well known that hydrophobic chemicals were not easily dissolved in water but easily dissolved in organic solvent. CPT was no exception, which could be dissolved in DMSO. Accordingly, single oil-in-water $(\mathrm{o} / \mathrm{w})$ emulsion technique was used to prepare CPT-loaded Ac- $\beta$ CD nanoparticles using DCM as solvent, DMSO as cosolvent for CPT, and water as dispersing agent, as illustrated in Figure 3(a). Formed nanoparticles exhibited nearly spherical structure, confirmed by SEM image (Figure 3(b)). Statistic 


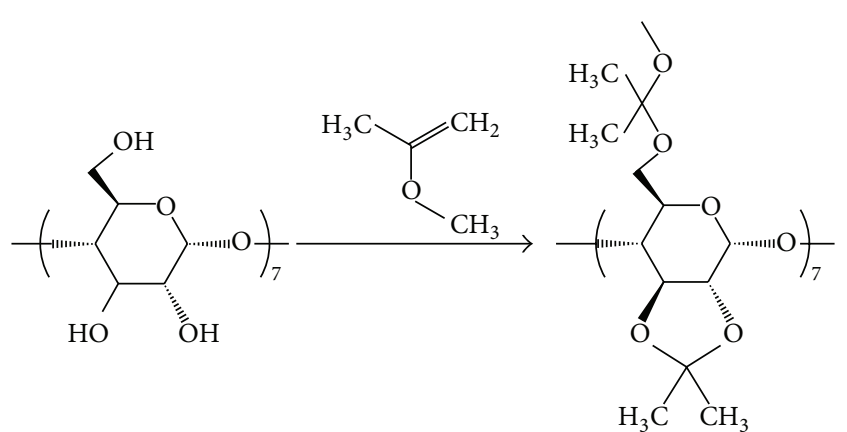

(a)

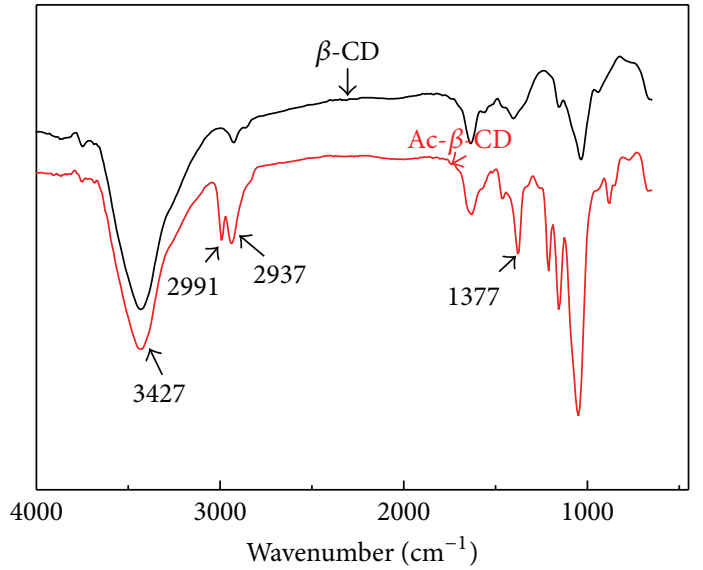

(b)

FIgURE 2: (a) Schematic illustration to show the formation and molecular structures of Ac- $\beta$-CD. (b) IR spectra of CD and its derivative Ac- $\beta$-CD.

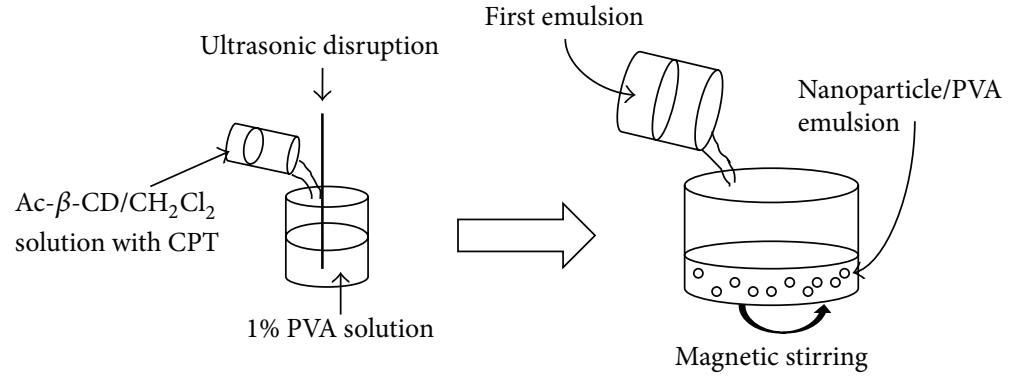

(a)

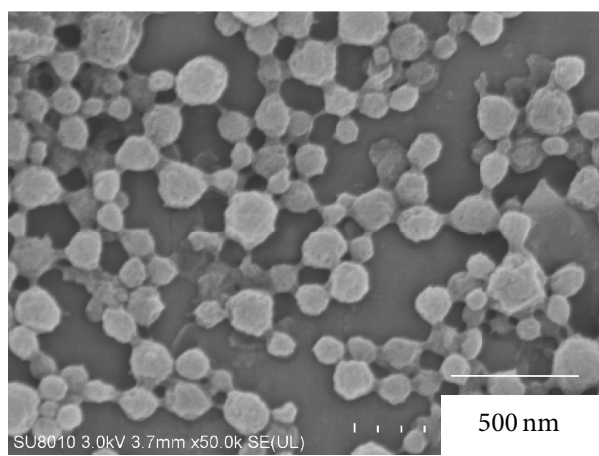

(b)

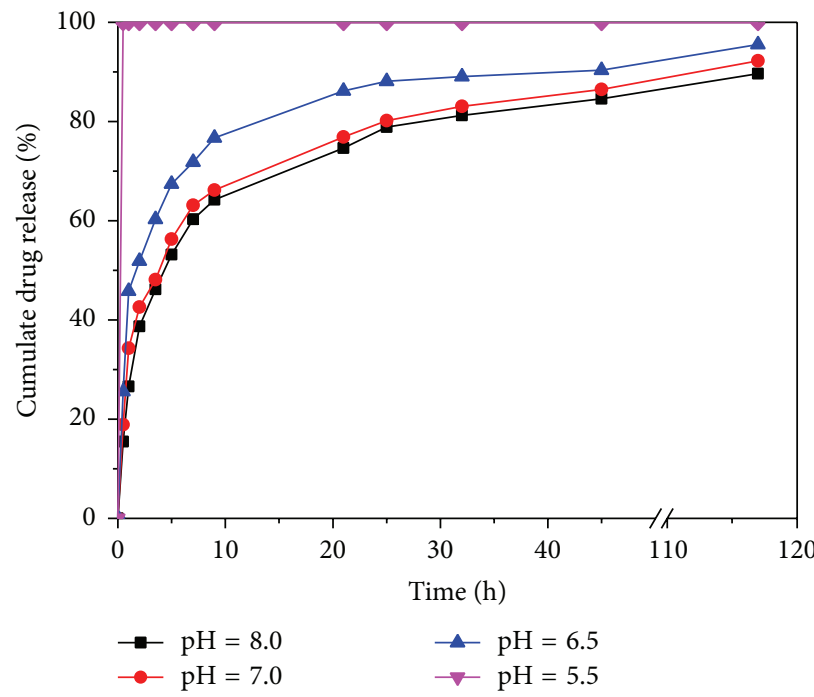

(c)

FIGURE 3: (a) Schematic illustration to show the formation of nanoparticle solution. (b) SEM image of CPT-loaded Ac- $\beta$-CD nanoparticles. (c) Cumulate $\mathrm{CPT}$ release behaviors in different $\mathrm{pH}$ value medium at $37^{\circ} \mathrm{C}$. 


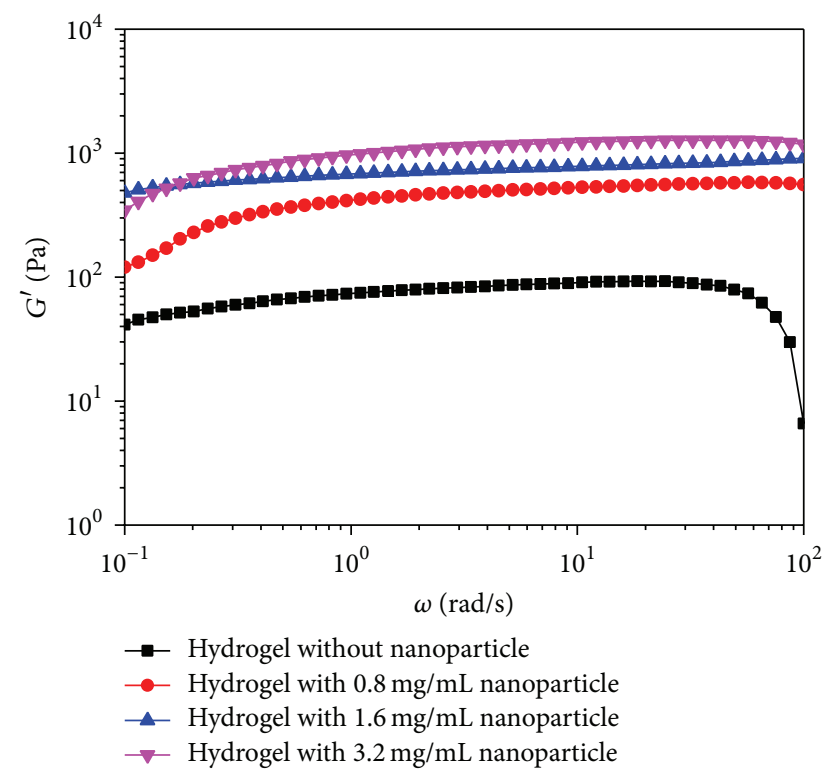

(a)

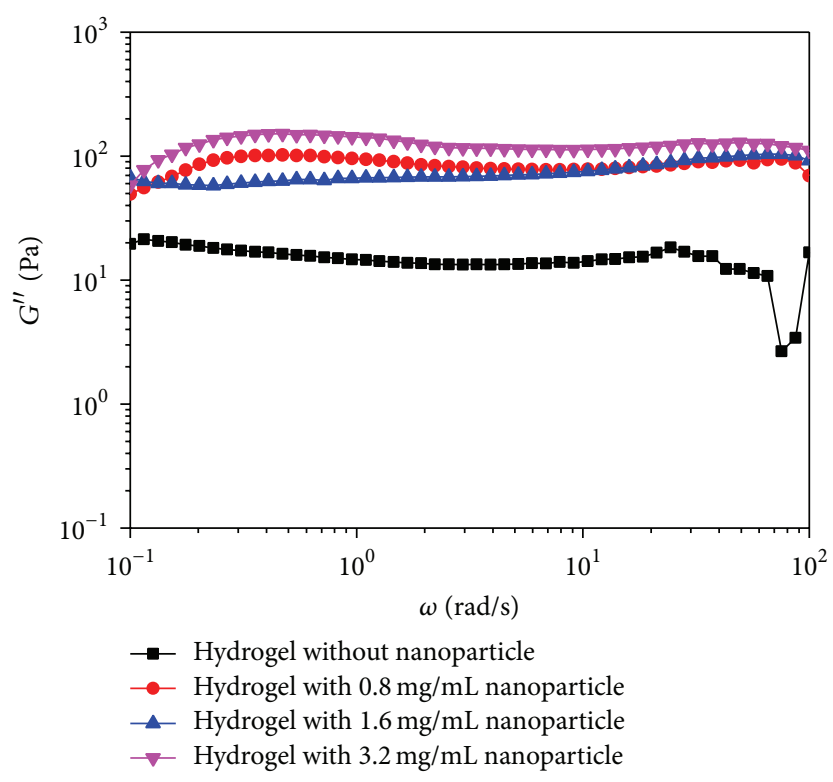

(b)

FIGURE 4: Storage modulus (a) and loss modulus (b) of different hydrogel as a function of compressing frequency $(\omega)$. Strain and temperature were set at $1 \%$ and $37^{\circ} \mathrm{C}$, respectively.

diameter of CPT-loaded Ac- $\beta$-CD nanoparticles was $209 \pm$ $40 \mathrm{~nm}$ from Figure 3(b).

Further, in order to examine properties of the drug carrier, CPT release behaviors in different $\mathrm{pH}$ value medium were characterized in Figure 3(c) and described as follows. In neutral and mild alkaline solution $(\mathrm{pH}=7.0$ and 8.0), $30 \%$ CPT was burst released from nanoparticles at first. Then, CPT was slowly released from nanoparticles in the following $30-40 \mathrm{~h}$. At last, the remaining $10-20 \%$ CPT was gradually released from nanoparticles in the next 4 days. The first burst release comes from drug entrapped on the surface of nanoparticle, which was easily dissolved in the released medium. Theoretically, the following slow release is due to gradual diffusion of drug from nanoparticles interior to outside released solution. Similarly, $45 \%$ CPT was burst released from nanoparticles in $\mathrm{pH}=6.5$ solution, the following slow release lasted $10-20 \mathrm{~h}$ until $80-85 \%$ CPT was released from nanoparticle, and the last gradual release in the next 4 days only included $10 \%$ CPT. The difference of CPT release profile in $\mathrm{pH}>7$ medium versus in $\mathrm{pH}=6.5$ medium might be ascribed to slight and slow dissolution of Ac- $\beta$ $\mathrm{CD}$ nanoparticles. But in mild acidic medium $(\mathrm{pH}=5.5)$, the condition was different. All CPT was fast released from nanoparticles at the beginning. Undeniably, the fast and burst release behavior in mild acidic solution is a result of fast dissolution of Ac- $\beta-C D$ nanoparticles, which is consistent with the $\mathrm{pH}$-responsive property of Ac- $\beta$-CD. In short, Ac$\beta$-CD nanoparticles displayed $\mathrm{pH}$ dependent changes due to hydrolysis of Ac- $\beta-C D$, verified by all the above-mentioned results.

3.2. Injectable Hydrogel. Inspired by previous report of GO hydrogel using PVA as a physical cross-linking agent, we designed $\mathrm{pH}$-sensitive injectable nanoparticle composite hydrogel based on the mixture of GO solution and CPTloaded Ac- $\beta$-CD nanoparticles/PVA solution $[15,17,18]$. It was reported that the optimum content ratio $\left(r_{\mathrm{PVA} / \mathrm{GO}}\right)$ of GO hydrogel was between $1: 2$ and 1:10, and the optimum PVA concentration before mixture was from $1.5 \mathrm{mg} / \mathrm{mL}$ to $7.5 \mathrm{mg} / \mathrm{mL}$ [15]. Since the PVA concentration of preformed nanoparticles solution was measured at $2.8 \mathrm{mg} / \mathrm{mL}$, the solution acted as one hydrogel precursor directly. According to the above-mentioned report, the concentration of GO solution, the other hydrogel precursor, was fixed at $7.8 \mathrm{mg} / \mathrm{mL}$ in the work. The influence of Ac- $\beta$-CD nanoparticle on the properties of injectable hydrogel was investigated as follows. All hydrogels formed within $1 \mathrm{~min}$ immediately after the two solutions were mixed under violently shaking condition regardless of Ac- $\beta$-CD nanoparticle concentration, which indicated that Ac- $\beta-C D$ nanoparticle had little effect on the gelation of hydrogel.

Since dynamic viscoelasticity was a kind of means to have an insight into the inner structure of polymer materials, the viscoelastic behaviors of formed hydrogels were tracked by the storage moduli and loss moduli as a function of compressing frequency and were shown in Figure 4. On the whole, both storage modulus (Figure 4(a)) and loss modulus (Figure $4(\mathrm{~b})$ ) of hydrogels showed slight stable frequency dependence according to Ac- $\beta$-CD nanoparticle concentration over a frequency range of $10^{-1}-10^{2} \mathrm{rad} / \mathrm{s}$, which reflected the homogeneous and stable inner structure of the hydrogels, especially under low frequency. Simultaneously, it was found by comparing Figures 3(a) and 3(b) that the storage moduli of all hydrogels were nearly 3-7 times higher than the loss moduli, which indicated that these hydrogels were elastomeric. More importantly, the influence of Ac- $\beta$-CD nanoparticle on the strength of hydrogel was also displayed in Figure 4. The storage modulus increased with the increase 


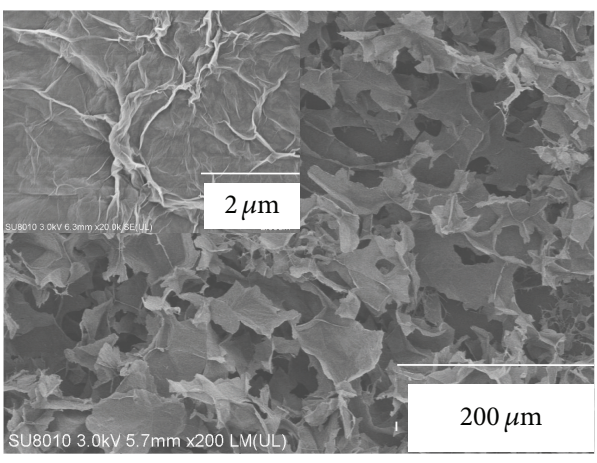

(a)

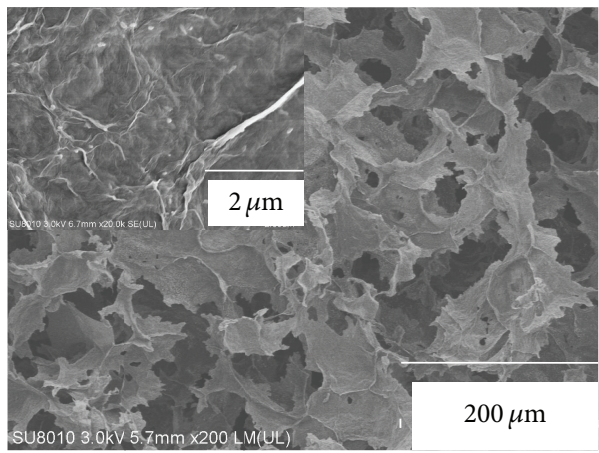

(c)

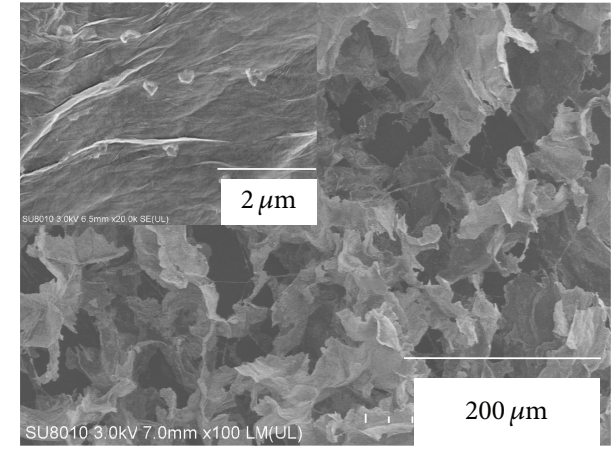

(b)

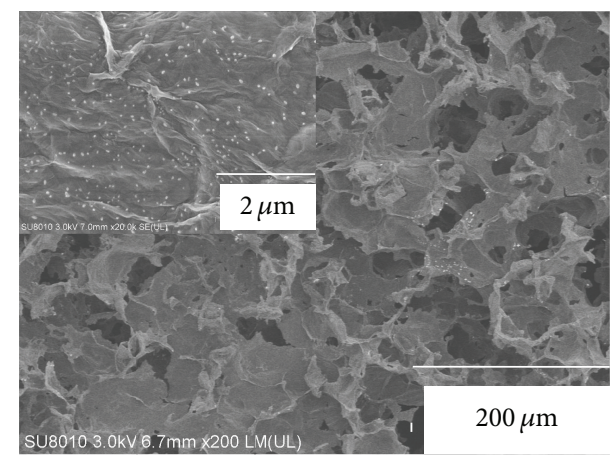

(d)

FIGURE 5: SEM images of different hydrogel as a function of nanoparticle concentration: (a) hydrogel without nanoparticle, (b) hydrogel with $0.8 \mathrm{mg} / \mathrm{mL}$ nanoparticle, (c) hydrogel with $1.6 \mathrm{mg} / \mathrm{mL}$ nanoparticle, and (d) hydrogel with $3.2 \mathrm{mg} / \mathrm{mL}$ nanoparticle.

of Ac- $\beta$-CD nanoparticle concentration when nanoparticle concentration was lower than $1.6 \mathrm{mg} / \mathrm{mL}$. Hydrogel with nanoparticles also had larger loss moduli than hydrogel without nanoparticles. These differences might be ascribed to the potentiation of homogeneous distributed Ac- $\beta$-CD nanoparticles in hydrogel. Moreover, the storage modulus of hydrogel without nanoparticles showed some decrease at high compressing frequency (over $40 \mathrm{rad} / \mathrm{s}$ ), and the loss modulus of that also showed some instability. But the same phenomenon did not emerge in hydrogel with nanoparticles, which indicated that hydrogel with nanoparticles possessed more stable physical cross-linking structure than hydrogel without nanoparticles. Thus, it was inferred that Ac$\beta$-CD nanoparticles could help to enhance the physical cross-linking points though the "supergelator" of injectable hydrogel mainly came from the supermolecular interactions between GO sheets and PVA chain.

In order to have further insight of microscopic structure, hydrogels were lyophilized for morphological study in Figure 5. All hydrogels exhibited the typical porous cross-linked structure of lyophilized hydrogel. Also, for all hydrogels, typical wrinkled structure of GO was easily found in further amplification of SEM images. But differently, the porous cross-linked structure became more homogeneous with smaller pore size and less debris when nanoparticles concentration was higher. This might be attributed to the potentiation of nanoparticle, which increased and enhanced cross-linking sites. Additionally, some nanoparticles in the
GO surface were scattered in the hydrogel with nanoparticles and increased with the increase of nanoparticle concentration. Moreover, no obvious two-phase structure was observed from these SEM images, revealing that PVA chains were dispersed on the GO sheets without phase separation on a large scale.

Besides the above-mentioned structural characteristic, the $\mathrm{pH}$ dependent property was also detected. All hydrogels were in gel state in acid and neutral environment and underwent a reversible sol-gel transition in alkaline environment just as previous report of GO/PVA self-assembling hydrogel [15]. Therefore, owing to mild acid environment of tumor tissue, they could aggregate to tumor site stably once they were injected into lesion tissues. That is, these hydrogels were ideal carriers for anticancer drug delivery.

Finally, in vitro drug release study was performed to evaluate the drug releasing ability of hydrogel. Considering encapsulated drug amount, we chose hydrogel with $3.2 \mathrm{mg} / \mathrm{mL}$ nanoparticle for the next drug release study. Figure 6 plots the drug release profiles of these hydrogels in PBS with different $\mathrm{pH}$ value. 10\% CPT was burst released from hydrogel in any medium regardless of $\mathrm{pH}$ value at first. Then, $40 \%$ CPT was gradually released in $\mathrm{pH}=7.5$ solution in $20 \mathrm{~h}$, and only $15-20 \%$ was gradually released in $\mathrm{pH}=5.5$ solution in the same time period, but little released CPT was detected in $\mathrm{pH}=6.5$ and/or 6.0 solution. In the final $4 \mathrm{~d}$ detection, no more CPT was released from hydrogel. These results were ascribed to dual carriers, namely, Ac- $\beta$-CD 


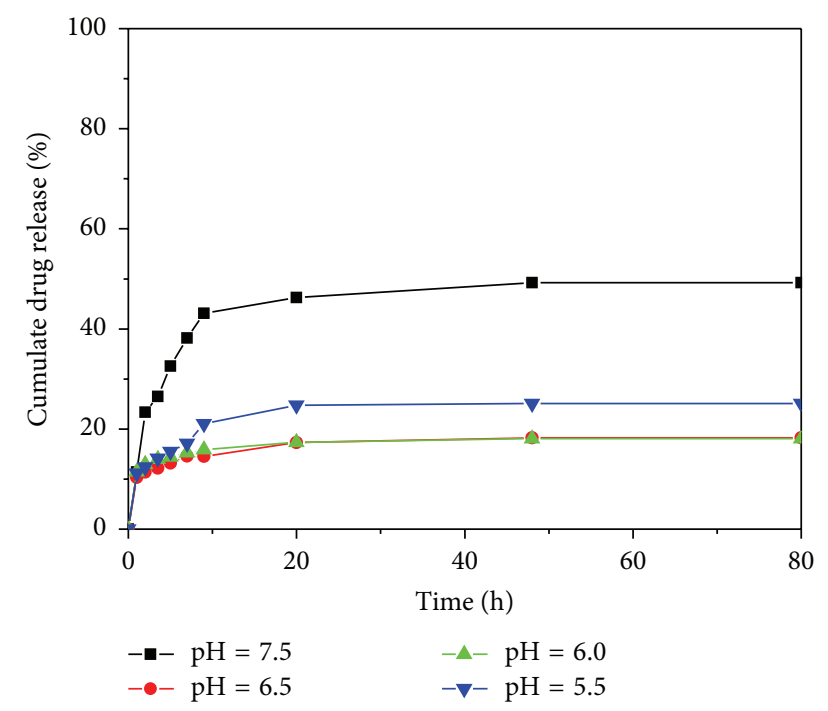

Figure 6: Cumulate CPT release behaviors of injectable hydrogel in different medium at $37^{\circ} \mathrm{C}$.

nanoparticle and GO sheets, in hydrogel. Besides the abovementioned $\mathrm{pH}$ dependent drug controlling effect of Ac- $\beta-\mathrm{CD}$ nanoparticle, GO sheets play an important role in controlling drug release for the injectable hydrogel. It had been verified in our previous research that GO sheets had strong interactions with CPT molecules that formed a barrier for CPT diffusion outside hydrogel. In mild alkaline environment, GO sheets were in negatively charged state on account of plenty of hydroxyl, epoxy, and carboxyl groups on the surface of GO sheets, resulting in decreased interactions between sheets and molecules. Therefore, some of the released CPT from Ac$\beta$-CD nanoparticles could gradually diffuse from hydrogel. While in mild acid environment, nonion state of GO sheet might increase the attraction of CPT molecule, resulting in less CPT release. Moreover, a little more drug release amount might be attributed to combined action of fast hydrolysis of Ac- $\beta$-CD nanoparticles and strong attraction of GO sheets. On the whole, the feature ensured anticancer drug staying in lesions location.

\section{Conclusion}

Injectable hydrogels incorporated with drug encapsulated Ac- $\beta$-CD nanoparticles were designed and prepared for delivery of anticancer drugs. First of all, Ac- $\beta-C D$, an acid-sensitive $\beta$-CD derivative, was successfully synthesized by acetonation under anhydrous oxygen-free condition. Secondly, CPT-loaded Ac- $\beta$-CD nanoparticles were easily obtained by single oil-in-water $(\mathrm{o} / \mathrm{w})$ emulsion technique. Formed nanoparticles exhibited nearly spherical structure with diameter of $209 \pm 40 \mathrm{~nm}$. The drug release behavior of nanoparticles displayed $\mathrm{pH}$ dependent changes due to hydrolysis of Ac- $\beta$-CD. Namely, relative gradual and sustained release in some period was detected in neutral and alkaline environment, and the fast and burst release was tracked in mild acidic solution. Thirdly, injectable hydrogels were assembled by supermolecular interaction between GO sheets and PVA chains in the process of simple mixture and their injectable property was confirmed by short gelation time and good mobility of two precursors. The dynamic mechanical properties of hydrogels and SEM images of lyophilized hydrogels reflected their homogeneous and stable cross-linked inner structure. At the same time, Ac- $\beta$-CD nanoparticles could help to enhance physical cross-linking and also had an effect on hydrogel inner morphology. Moreover, all hydrogels underwent a reversible sol-gel transition in alkaline environment. Finally, the results of in vitro drug release profile indicated that hydrogel could control drug release or bind drug inside depending on the $\mathrm{pH}$ value of released medium. All in all, this study will provide a simple approach to design and fabricate novel functional materials for anticancer delivery.

\section{Conflict of Interests}

The authors declare that there is no conflict of interests regarding the publication of this paper.

\section{Acknowledgment}

This study is financially supported by Qing Lan Project, the Natural Science Foundation of China (51103066).

\section{References}

[1] H. Chen, X. Liu, Y. Dou et al., "A pH-responsive cyclodextrinbased hybrid nanosystem as a nonviral vector for gene delivery," Biomaterials, vol. 34, no. 16, pp. 4159-4172, 2013.

[2] H. He, S. Chen, J. Zhou et al., "Cyclodextrin-derived pHresponsive nanoparticles for delivery of paclitaxel," Biomaterials, vol. 34, no. 21, pp. 5344-5358, 2013.

[3] Q. Shi, L. Zhang, M. Liu et al., "Reversion of multidrug resistance by a $\mathrm{pH}$-responsive cyclodextrin-derived nanomedicine in drug resistant cancer cells," Biomaterials, vol. 67, pp. 169-182, 2015.

[4] S. Khoee and R. Rahmatolahzadeh, "Synthesis and characterization of $\mathrm{pH}$-responsive and folated nanoparticles based on self-assembled brush-like PLGA/PEG/AEMA copolymer with targeted cancer therapy properties: a comprehensive kinetic study," European Journal of Medicinal Chemistry, vol. 50, pp. 416-427, 2012.

[5] Z. Zhou, A. Badkas, M. Stevenson, J. Y. Lee, and Y. K. Leung, "Herceptin conjugated PLGA-PHis-PEG pH sensitive nanoparticles for targeted and controlled drug delivery," International Journal of Pharmaceutics, vol. 487, no. 1-2, pp. 81-90, 2015.

[6] K. E. Broaders, J. A. Cohen, T. T. Beaudette, E. M. Bachelder, and J. M. J. Fréchet, "Acetalated dextran is a chemically and biologically tunable material for particulate immunotherapy," Proceedings of the National Academy of Sciences of the United States of America, vol. 106, no. 14, pp. 5497-5502, 2009.

[7] N. Kanthamneni, S. Sharma, S. A. Meenach et al., "Enhanced stability of horseradish peroxidase encapsulated in acetalated dextran microparticles stored outside cold chain conditions," International Journal of Pharmaceutics, vol. 431, no. 1-2, pp. 101110, 2012. 
[8] S. A. Meenach, Y. J. Kim, K. J. Kauffman, N. Kanthamneni, E. M. Bachelder, and K. M. Ainslie, "Synthesis, optimization, and characterization of camptothecin-loaded acetalated dextran porous microparticles for pulmonary delivery," Molecular Pharmaceutics, vol. 9, no. 2, pp. 290-298, 2012.

[9] S. Suarez, G. N. Grover, R. L. Braden, K. L. Christman, and A. Almutairi, "Tunable protein release from acetalated dextran microparticles: a platform for delivery of protein therapeutics to the heart post-MI," Biomacromolecules, vol. 14, no. 11, pp. 39273935, 2013.

[10] X. Hu, D. Li, F. Zhou, and C. Gao, "Biological hydrogel synthesized from hyaluronic acid, gelatin and chondroitin sulfate by click chemistry," Acta Biomaterialia, vol. 7, no. 4, pp. 1618-1626, 2011.

[11] H. Tan, X. Gao, J. Sun, C. Xiao, and X. Hu, "Double stimulusinduced stem cell aggregation during differentiation on a biopolymer hydrogel substrate," Chemical Communications, vol. 49, no. 98, pp. 11554-11556, 2013.

[12] H. P. Tan, C. Xiao, J. C. Sun, D. S. Xiong, and X. H. Hu, "Biological self-assembly of injectable hydrogel as cell scaffold via specific nucleobase pairing," Chemical Communications, vol. 48, no. 83, pp. 10289-10291, 2012.

[13] X. Hu, D. Li, H. Tan, C. Pan, and X. Chen, "Injectable graphene oxide/graphene composite supramolecular hydrogel for delivery of anti-cancer drugs," Journal of Macromolecular Science, Part A: Pure and Applied Chemistry, vol. 51, no. 4, pp. 378-384, 2014.

[14] H. Tan and K. G. Marra, "Injectable, biodegradable hydrogels for tissue engineering applications," Materials, vol. 3, no. 3, pp. 1746-1767, 2010.

[15] H. Bai, C. Li, X. Wang, and G. Shi, "A pH-sensitive graphene oxide composite hydrogel," Chemical Communications, vol. 46, no. 14, pp. 2376-2378, 2010.

[16] J.-H. Yang and Y.-D. Lee, "Highly electrically conductive rGO/PVA composites with a network dispersive nanostructure," Journal of Materials Chemistry, vol. 22, no. 17, pp. 85128517, 2012.

[17] R. K. Layek, A. K. Das, M. U. Park, N. H. Kim, and J. H. Lee, "Layer-structured graphene oxide/polyvinyl alcohol nanocomposites: dramatic enhancement of hydrogen gas barrier properties," Journal of Materials Chemistry A, vol. 2, no. 31, pp. 12158-12161, 2014.

[18] Y. F. Huang, M. Q. Zhang, and W. H. Ruan, "High-watercontent graphene oxide/polyvinyl alcohol hydrogel with excellent mechanical properties," Journal of Materials Chemistry A, vol. 2, no. 27, pp. 10508-10515, 2014.

[19] X. Hu, X. Ma, H. Tan, and D. Li, "Preparation of water-soluble and biocompatible graphene," Micro \& Nano Letters, vol. 8, no. 6, pp. 277-279, 2013.

[20] C. Huang, H. Bai, C. Li, and G. Shi, "A graphene oxide/hemoglobin composite hydrogel for enzymatic catalysis in organic solvents," Chemical Communications, vol. 47, no. 17, pp. 4962-4964, 2011.

[21] A. Sahu, W. I. Choi, and G. Tae, "A stimuli-sensitive injectable graphene oxide composite hydrogel," Chemical Communications, vol. 48, no. 44, pp. 5820-5822, 2012.

[22] J. Wu, A. Chen, M. Qin et al., "Hierarchical construction of a mechanically stable peptide-graphene oxide hybrid hydrogel for drug delivery and pulsatile triggered release in vivo," Nanoscale, vol. 7, no. 5, pp. 1655-1660, 2015.

[23] F. Du, H. Meng, K. Xu et al., "CPT loaded nanoparticles based on beta-cyclodextrin-grafted poly(ethylene glycol)/poly
(L-glutamic acid) diblock copolymer and their inclusion complexes with CPT," Colloids and Surfaces. B, Biointerfaces, vol. 113, pp. 230-236, 2013. 

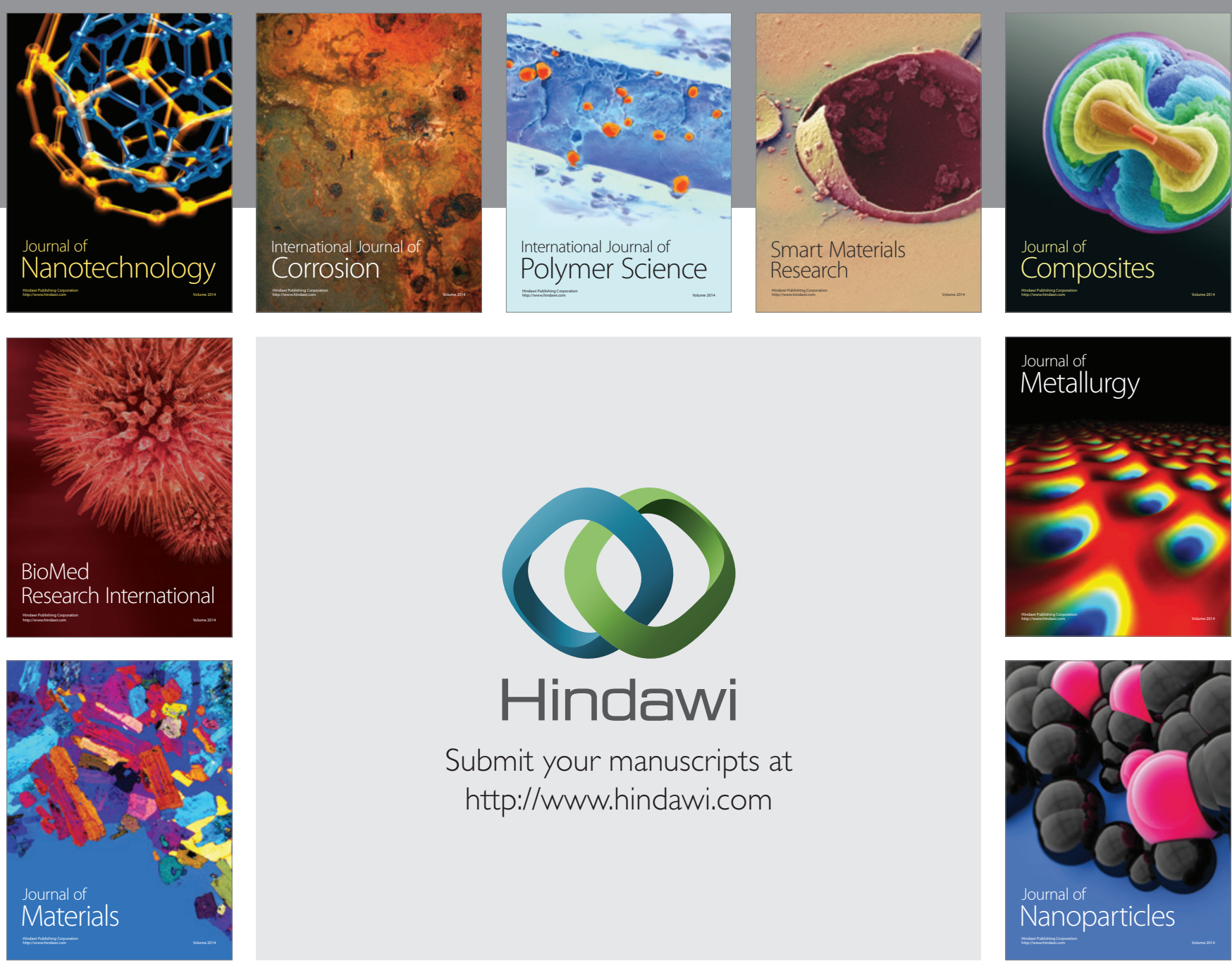

\section{Hindawi}

Submit your manuscripts at

http://www.hindawi.com

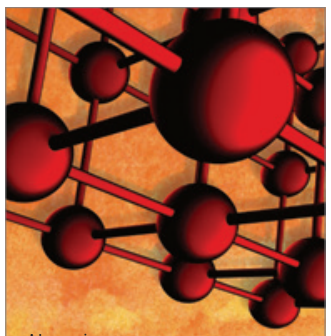

Materials Science and Engineering
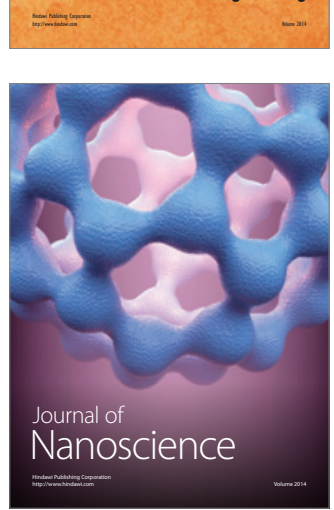
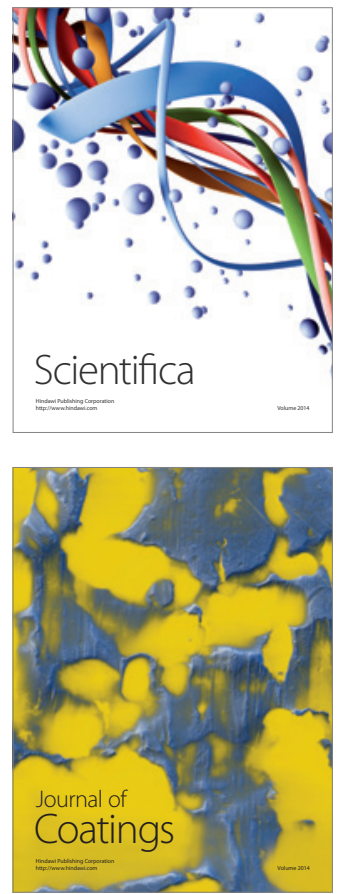
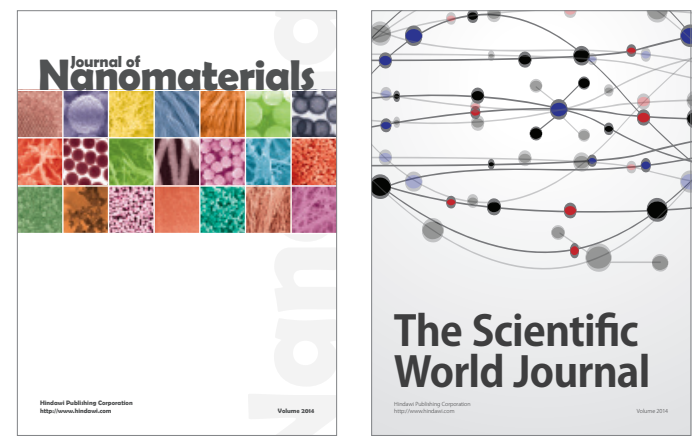

The Scientific World Journal
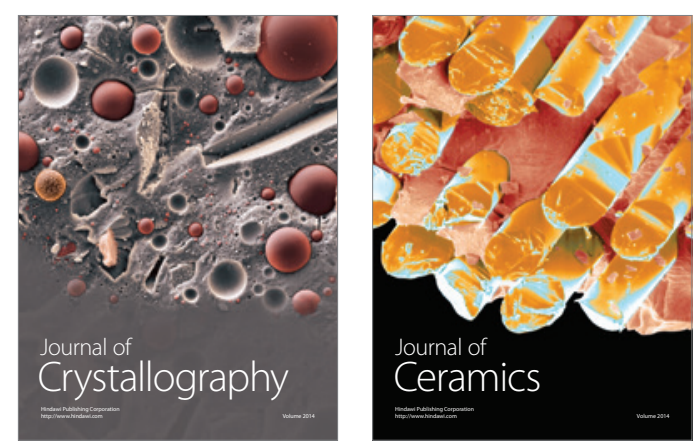
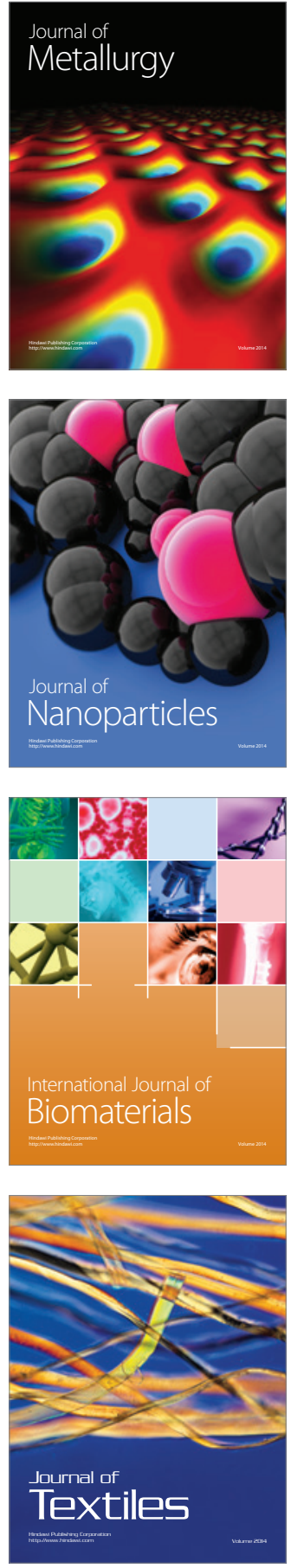